Gut and Liver, Vol. 14, No. 6, November 2020, pp. 685-698

\title{
Biologics for the Management of Inflammatory Bowel Disease: A Review in Tuberculosis-Endemic Countries
}

\author{
Rupa Banerjee ${ }^{1}$, Raja Affendi Raja $\mathrm{Ali}^{2}$, Shu Chen $\mathrm{Wei}^{3}$, and Shashi Adsul ${ }^{4}$ \\ ${ }^{1}$ IBD Center, Asian Institute of Gastroenterology, Hyderabad, India, ${ }^{2}$ Gastroenterology Unit, Department of Medicine, Faculty of Medicine, \\ The National University of Malaysia, Kuala Lumpur, Malaysia, ${ }^{3}$ Department of Internal Medicine, IBD Clinical and Basic Research Integrated \\ Center, National Taiwan University Hospital, National Taiwan University College of Medicine, Taipei, Taiwan, and ${ }^{4}$ Takeda Pharmaceuticals \\ International AG, Zurich, Switzerland
}

The advent of biologics and biologic therapy has transformed the management of inflammatory bowel disease (IBD) with enhanced early and adequate responses to treatment, fewer hospitalizations, a reduced need for surgery, and unprecedented outcomes including complete mucosal and histologic healing. However, an important issue with the use of anti-tumor necrosis factor (anti-TNF) agents in IBD is the increased risk of tuberculosis (TB). This is compounded by the diagnostic dilemma when differentiating between Crohn's disease and gastrointestinal TB, and the potentially serious consequences of initiating an incorrect treatment in the case of misdiagnosis. The interplay between IBD and TB is most relevant in Asia, where more than $60 \%$ of the 10.4 million new TB cases in 2016 were reported. A number of studies have reported an increased risk of TB with anti-TNF agents, including in patients who had tested negative for TB prior to treatment initiation. The limited evidence currently available regarding adhesion molecule antagonists such as vedolizumab suggests a comparatively lower risk of TB, thus making them a promising option for IBD management in TBendemic regions. This comprehensive review examines the available literature on the risk of TB with the use of biologics in the TB-endemic regions of Asia, focusing on the diagnostic dilemma, the risk of reactivation, and the optimized management algorithms for latent and active disease. (Gut Liver 2020;14:685-698)

Key Words: Colitis, ulcerative; Crohn disease; Biologic therapy; Tuberculosis; Asia

\section{INTRODUCTION}

Inflammatory bowel disease (IBD) is a group of chronic relapsing and remitting intestinal disorders that can be broadly categorized as either Crohn's disease (CD) or ulcerative colitis (UC). While CD and UC share some commonalities, there exist distinct clinical and pathological features differentiating the two disorders. ${ }^{1,2}$ The etiopathogenesis of IBD is still unknown; however, the interactions of immune dysfunction with genetic factors, environmental exposure and gut microbiome are thought to be heavily involved in these complex disorders. ${ }^{3}$

Management of IBD is primarily based on location and severity of the disease. Accordingly, numerous classification systems have been used. The Montreal classification (see Table 1) categorizes $\mathrm{CD}$ by age at diagnosis, location and behavior, and categorizes UC by disease extent and the severity of relapse., Disease severity in CD is defined by disease activity and is classified into mild (Crohn's Disease Activity Index [CDAI] score of 150 to 220), moderate to severe (CDAI 220 to 450) and severe/ fulminant (CDAI >450). A CDAI score less than 150 signifies that the disease is in remission. ${ }^{6}$

The 3rd European Crohn's and Colitis Organisation (ECCO) guidelines for UC classify UC disease severity into mild, moderate and severe, according to the Truelove-Witts Index.

Remission in UC is defined by fewer than 4 stools/day with no bleeding and no mucosal lesions at endoscopy. ${ }^{7}$

The standard treatment for IBD has included mesalamine, steroids and immunomodulators. In recent years, the management has evolved substantially following the introduction of antitumor necrosis factor (anti-TNF) agents. ${ }^{8}$ Although efficacious, the increased risk of tuberculosis (TB) with anti-TNF agents remains a concern. ${ }^{9}$ The objective of this review is to discuss the

Correspondence to: Rupa Banerjee

IBD Center, Asian Institute of Gastroenterology, AIG Hospitals, Mindspace Road, Gachibowli, Hyderabad 500032, India

Tel: +91-9849287530, Fax: +91-40-2332-4255, E-mail: dr_rupa_banerjee@hotmail.com

Received on June 17, 2019. Revised on November 1, 2019. Accepted on November 25, 2019.

pISSN 1976-2283 eISSN 2005-1212 https://doi.org/10.5009/gnl19209

@ This is an Open Access article distributed under the terms of the Creative Commons Attribution Non-Commercial License (http://creativecommons.org/licenses/by-nc/4.0) which permits unrestricted non-commercial use, distribution, and reproduction in any medium, provided the original work is properly cited. 
Table 1. The Montreal Classification of CD and UC

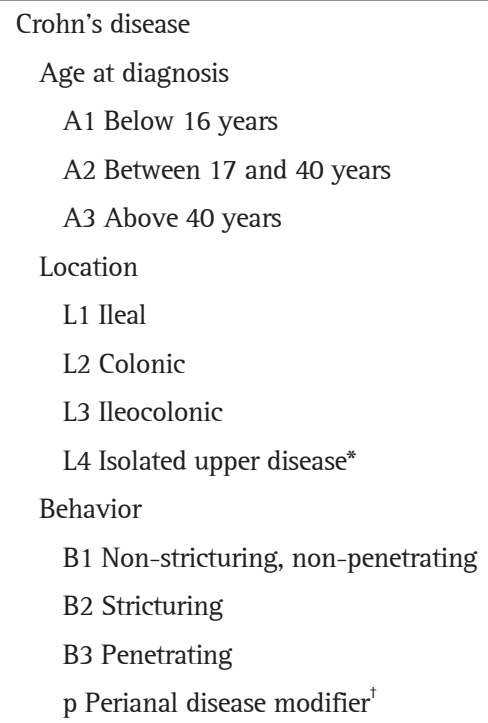

Ulcerative colitis

Extent

E1 Ulcerative proctitis: involvement limited to the rectum (that is, proximal extent of inflammation is distal to the rectosigmoid junction)

E2 Left-sided UC (distal UC): involvement limited to a proportion of the colorectum distal to the splenic flexure

E3 Extensive UC (pancolitis): involvement extends proximal to the splenic flexure

\section{Severity}

So Clinical remission: asymptomatic

S1 Mild UC: passage of 4 or fewer stools/day (with or without blood), absence of any systemic illness, and normal inflammatory markers (ESR)

S2 Moderate UC: passage of more than 4 stools per day but with minimal signs of systemic toxicity

S3 Severe UC: passage of at least 6 bloody stools daily, pulse rate of at least 90 beats per minute, temperature of at least $37.5^{\circ} \mathrm{C}$, hemoglobin of less than $10.5 \mathrm{~g} / 100 \mathrm{~mL}$, and ESR of at least $30 \mathrm{~mm} / \mathrm{hr}$

$\mathrm{CD}$, Crohn's disease; UC, ulcerative colitis; ESR, erythrocyte sedimentation rate.

${ }^{*} \mathrm{~L} 4$ is a modifier that can be added to L1-L3 when concomitant upper gastrointestinal disease is present; ${ }^{\dagger} \mathrm{p}$ is added to B1-B3 when concomitant perianal disease is present.

interplay between biologics and TB in patients with IBD.

For this review, a non-systematic review of the literature was conducted using keyword-based searches in PubMed and the Cochrane Library, supplemented by pragmatic searches. The following keywords were used-Crohn's disease, ulcerative colitis, inflammatory bowel disease, biologics, anti-TNF, adhesion molecule antagonists, anti-integrin agents, anti-interleukins (IL), tuberculosis, latent tuberculosis infection (LTBI), screening, and Asia. Guidelines published by the following organizations were reviewed-ECCO, the National Institute for Health and Care Excellence (NICE), the British Thoracic Society (BTS), the Taiwan Society of Inflammatory Bowel Disease (TSIBD), Asian Organization for Crohn's and Colitis (AOCC) and Asia Pacific Association of Gastroenterology (APAGE).

\section{MANAGEMENT OF IBD}

A variety of medical treatment options are currently available for IBD, and they vary by the disease site, disease activity (mild/ moderate/severe) and disease status (symptomatic or in remission). ${ }^{10}$

Conventional medical treatments for IBD include 5-aminosalicyclic acid (5-ASA/aminosalicylates/ mesalamine), corticosteroids and immunomodulators. Aminosalicylates are used in UC to induce remission in mild disease and mainly to maintain remission in oral or topical form. Corticosteroids are the mainstay of therapy for flares in IBD, to induce remission in moderate to severe disease. Oral beclomethasone dipropionate is an alternative to conventional steroids for moderate disease to induce remission. Immunomodulators such as azathioprine, mercaptopurine and methotrexate are useful adjunct treatments, and they are safer and better tolerated alternatives to long-term steroid therapy. Monotherapy with azathioprine or mercaptopurine is also used for the maintenance of remission in UC and CD. In acute severe UC, intravenous corticosteroids are recommended. For those who fail to respond to intravenous steroids, conventional options are intravenous ciclosporin and/or surgery. For patients with moderate to severe IBD not responsive 
to conventional therapy or with extensive small bowel involvement in $\mathrm{CD}$, biologics are recommended for effective treatment (NICE 2012; $;^{11}$ NICE 2013 $;^{12}$ 3rd ECCO guidelines for CD and $\left.\mathrm{UC}^{13,14}\right)$.

The aim of therapy in IBD today is inducing clinical remission, maintaining steroid-free remission and ensuring mucosal healing. Corticosteroids fail to induce remission in $~ 20 \%$ of patients, and more than $20 \%$ of patients become steroid-dependent. ${ }^{10,15}$ Biologics are the mainstay therapy of managing these patients. In severe acute UC and complicated $\mathrm{CD}$, biologics have an important role to play today. In the conventional "step-up" approach, the focus is on initiating treatment with medications associated with a lower risk of severe side effects. In this approach, immunomodulators and biologics are only considered in patients who are steroid-refractory or steroid-dependent. In the "top-down" approach, biologics and immunomodulators are introduced after diagnosis as a first-line therapy. This approach appears to be based on the potential disease-modifying effect of early intervention with biologics. Another alternative is an "accelerated step-up" approach which involves early or immediate start of immunomodulators along with conventional step-up therapy. ${ }^{16}$

Current approved biologic treatments in IBD include the following: ${ }^{1718}$ four anti-TNF agents (infliximab, adalimumab, golimumab, and certolizumab) and two adhesion molecule antagonists (natalizumab and vedolizumab). Additionally, ustekinumab has been approved for CD and an oral Janus kinase (JAK) inhibitor, tofacitinib, has been recently approved by the U.S. Food and Drug Administration (USFDA) for UC. ${ }^{19}$

A key issue with anti-TNF therapies is the development of primary nonresponse (i.e., lack of response to induction treatment) and secondary nonresponse (i.e., initial response during induction treatment followed by loss of response during maintenance treatment). Approximately 40\% of IBD patients may fail to achieve remission with biologics using the "top-down" approach. ${ }^{16}$ Other issues include the risk of serious side effects such as opportunistic infections, including $\mathrm{TB},{ }^{20,21}$ and potential development of neoplasia such as lymphoma or non-malignant skin cancer when used in combination with immunomodulators. $^{22}$

\section{TB, IBD AND BIOLOGICS}

The increasing use of biologics has brought into focus the interplay between TB and biologics in IBD. TB is relevant in IBD from the view of differential diagnosis, screening and treatment strategy. $^{23-25}$

\section{Diagnosis of IBD}

Due to the similarities between $\mathrm{CD}$ and gastrointestinal $\mathrm{TB}$ (giTB), differentiating between the two diseases can be challenging: (1) There exists considerable resemblance in clinical, radio- logical, endoscopic, surgical and histological features of CD and giTB (which typically occurs at the ileocecal region); (2) CD and giTB are both granulomatous conditions that can involve any part of the gastrointestinal tract; (3) There is no simple test that can be used to reliably differentiate CD from giTB.

These problems are compounded by the emergence of multidrug resistant TB (MDR-TB). In case of treatment-sensitive TB, the presence of a clear response to empirical anti-TB treatment (ATT) is often helpful in confirming the final diagnosis of TB. However, in case of MDR-TB, the therapeutic response to ATT is attenuated, thus complicating the issue.

Any error in making a diagnosis between CD and giTB can have potentially serious consequences. If $\mathrm{CD}$ is misdiagnosed as $\mathrm{TB}$, then the unnecessary ATT delivered may be harmful to the patient, and can result in delay in the treatment of the primary condition, i.e. CD. Indeed, empirical ATT has been identified as the single largest factor contributing to diagnostic delay in $\mathrm{CD}$ in TB-endemic countries. ${ }^{26}$ The reverse misdiagnosis is potentially more dangerous-if TB is misdiagnosed as $\mathrm{CD}$, then treatment with steroids or even biologics alone (for $\mathrm{CD}$ ) can result in an even further immunosuppressed status and disseminated TB, which can potentially be disastrous for the patient.

\section{Treatment of IBD}

With the increasing use of biologics, another area of importance is the risk of reactivation of TB following treatment with biologics.

Exposure to Mycobacterium tuberculosis may result in a latent infection that can reactivate later in life. LTBI refers to the persistence of live $M$. tuberculosis in patients who are not identifiable through any clinical symptom of active disease. In this case, it is assumed that the latent infection is insufficient to cause active disease except in response to a change in the immune status of the patient, e.g. due to use of immunosuppressive medications such as anti-TNF agents or due to advancing age $^{27}$ Of those with LTBI, approximately 10\% develop active TB over their lifetime, ${ }^{28}$ with an annual rate of reactivation being approximately $0.1 \%$ to $0.15 \% .^{29,30}$

$\mathrm{TNF}-\alpha$ is a key component of the immune response. In case of TB infection, the presence of TNF- $\alpha$ results in macrophage activation, cell recruitment, granuloma formation, and maintenance of granuloma integrity. ${ }^{31}$ In patients with LTBI, inhibition of TNF- $\alpha$ can result in a higher incidence of active TB infection, with clinical manifestations often similar to those seen in immune-compromised patients, i.e. atypical, miliary or extrapulmonary TB. This increased risk of TB may occur due to an impact on cell-mediated immunity, as anti-TNF agents can result in a reduction of CD8+ cells responsible for antimicrobial activity against $M$. tuberculosis. ${ }^{32}$ Another hypothesis for the increased risk of TB involves phagosome maturation. TNF- $\alpha$ leads to increase in maturation of phagosomes containing $M$. tuberculosis, and anti-TNF agents suppress phagosome maturation, 
resulting in impaired host response to mycobacteria. ${ }^{33}$

\section{Tuberculosis-endemic countries}

Understandably, all these issues related to the use of biologics in IBD are of greater concern in TB-endemic countries. TB is a major public health problem, and was one of the top 10 causes of death worldwide in 2016. According to recent estimates, ${ }^{34}$ there were an estimated 10.4 million new TB cases worldwide in 2016. Of these, $64 \%$ of the male cases and 59\% of the female cases were in Asia. India, Indonesia, China, the Philippines and Pakistan accounted for 56\% of all new cases. The 20 high burden countries based on the absolute number of incident cases are Angola, Bangladesh, Brazil, China, North Korea, Congo, Ethiopia, India, Indonesia, Kenya, Mozambique, Myanmar, Nigeria, Pakistan, Philippines, Russia, South Africa, Thailand, Tanzania and Vietnam. As seen in Fig. 1, the incidence of TB is high in almost all countries in the Asia-Pacific region. ${ }^{34}$

\section{Tests for TB screening and for diagnosis of LTBI}

Although a number of investigations are available for the diagnosis of $\mathrm{CD}$ and $\mathrm{TB}$, it is very difficult to differentiate $\mathrm{CD}$ from giTB based on clinical index of suspicion in an individual patient. ${ }^{24}$ Routine TB tests such as acid-fast bacilli (AFB) smear examination with Ziehl-Neelsen stain and conventional AFB culture are either low in sensitivity or time-consuming. More rapid and sensitive techniques (e.g., fluorescence technique for smear examination and Bactec technique for culture) are now available; however, the sensitivity of all of these instruments is rather poor in TB as it is a paucibacillary disease.

The diagnosis of LTBI is difficult, especially in immunocompromised patients. The tuberculin skin test (TST) and interferongamma release assays (IGRA) are the most pragmatic screening measures for TB infection. ${ }^{9}$ TST is an in vivo test that measures PPD-specific cell-mediated immunity, while IGRA are in vitro tests based on the rapid production of interferon-gamma by circulating mononuclear cells in response to M. tuberculosisspecific antigens. ${ }^{35}$ TST involves induction of a delayed-type hypersensitivity response following an intradermal injection of purified protein derivative. An induration $>5 \mathrm{~mm}$ seen at 48 to 72 hours after the injection is generally considered to be a positive reaction. The estimation of the risk of developing TB depends on various factors such as age, Bacillus CalmetteGuérin (BCG)-vaccination, and immune suppression diseases. The specificity of TST decreases if there has been exposure to nontuberculous mycobacteria and BCG vaccination; though, for BCG vaccinations given in infancy, their impact on TST is limited after 10 years or more. Overall, TST may be considered inadequate for assessing LTBI in BCG-vaccinated individuals. ${ }^{35}$

Whilst IGRA does not have cross-reactivity with BCG, neither IGRA nor TST are sufficiently sensitive. ${ }^{36}$ The sensitivity for detecting the presence of TB is higher with IGRA (78\% to 92\%) than with the TST (65\% to $77 \%$ ); however, the current level of sensitivity of IGRA is considered insufficient to confidently rule out active TB. ${ }^{35}$

An important consideration in the selection of tests for TB screening is the usage BCG vaccination in the region. The speci-

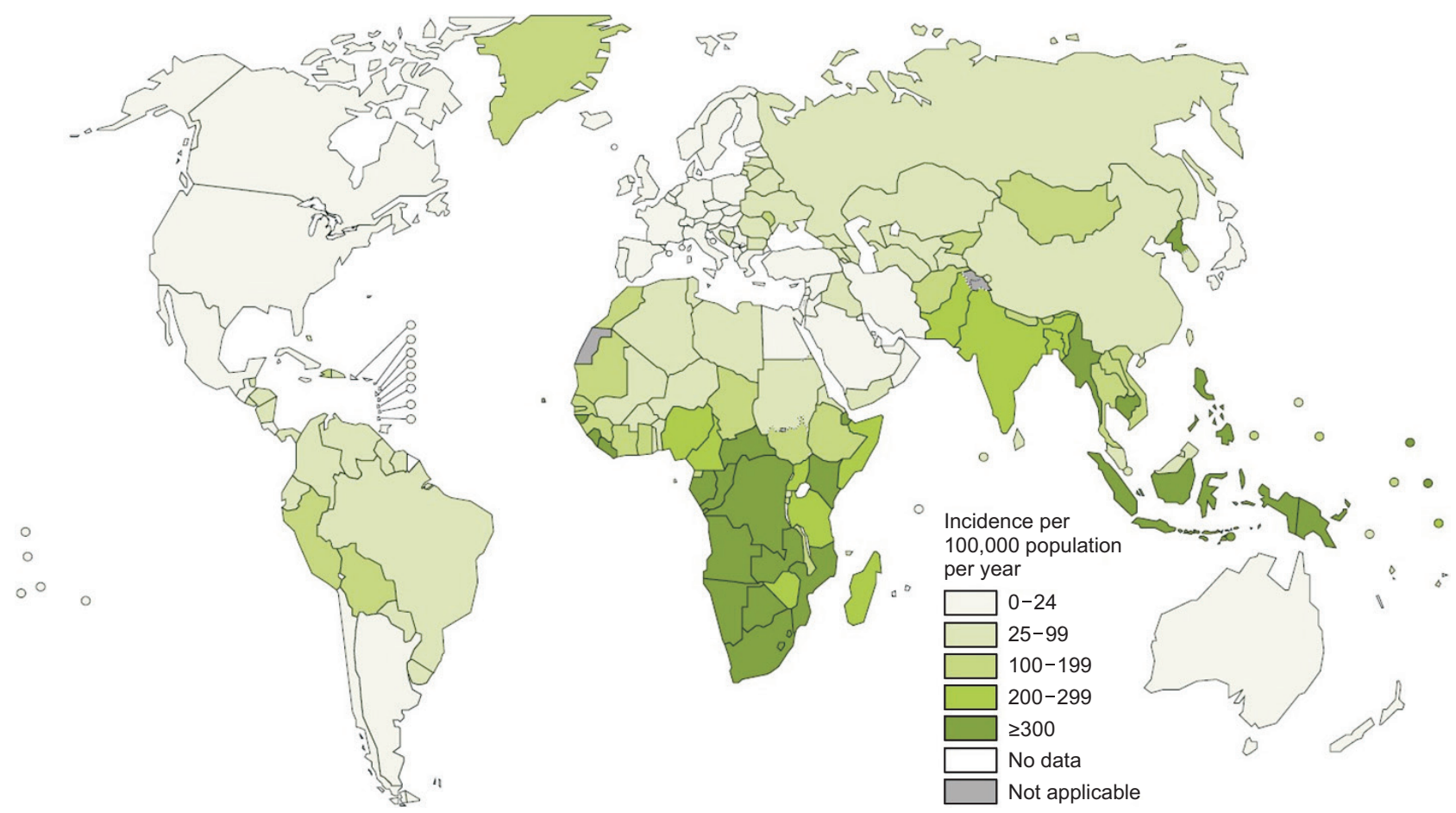

Fig. 1. Estimated tuberculosis (TB) incidence rates in 2016. Most countries in the Asia-Pacific region report TB incidences $\geq 100$ per 100,000 population per year. Figure source: World Health Organization (WHO). Global Tuberculosis Report 2017. ${ }^{34}$ 


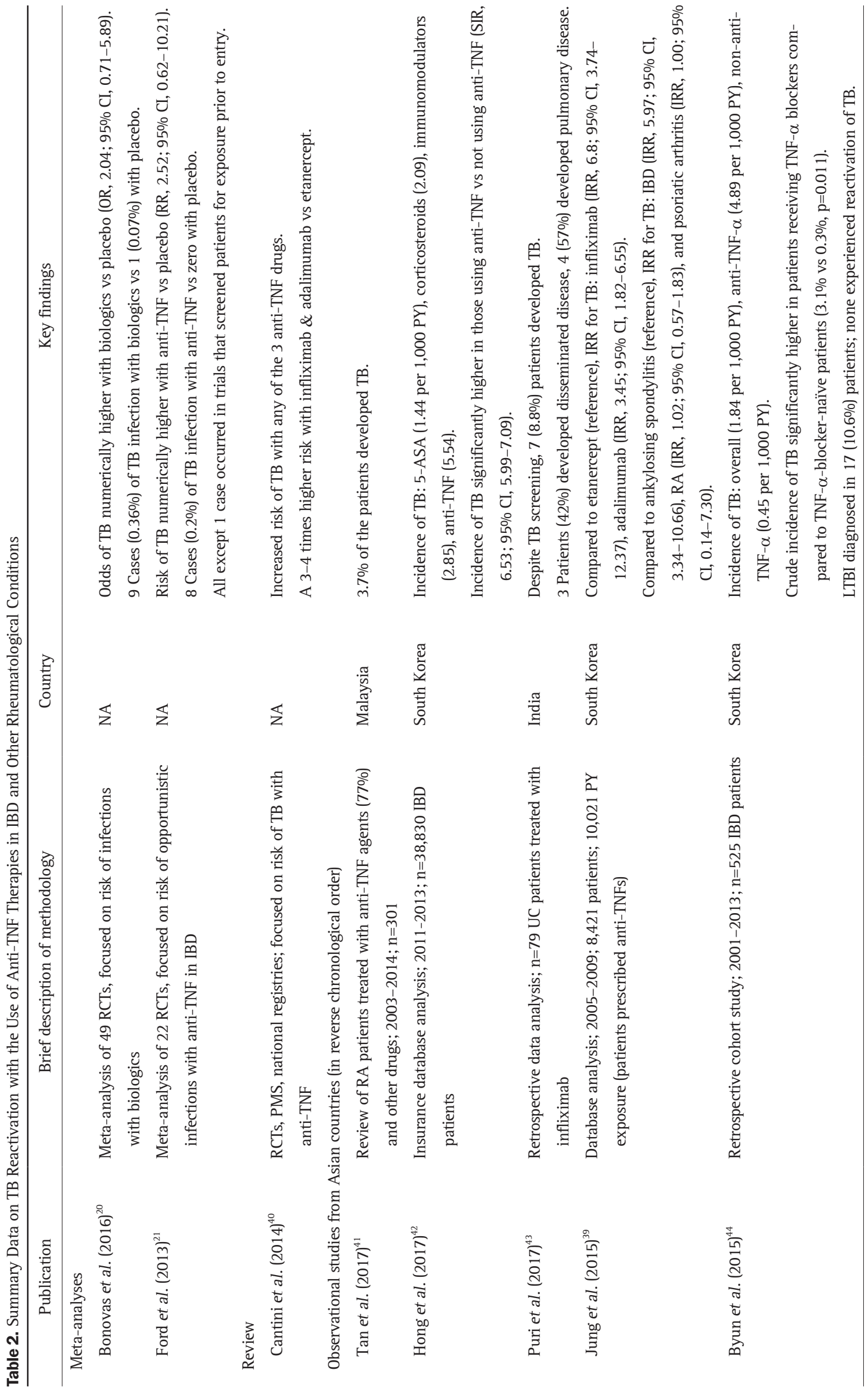




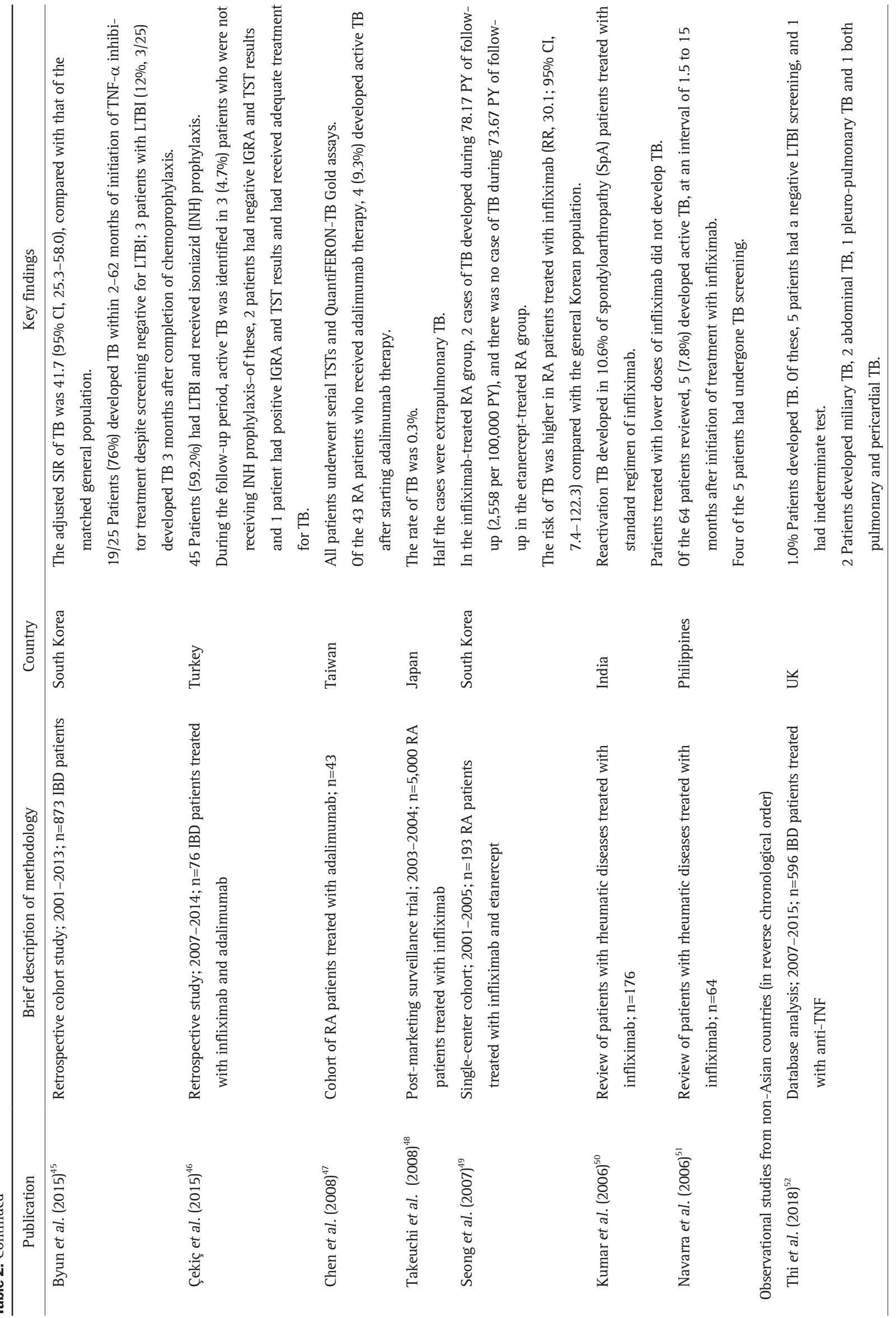




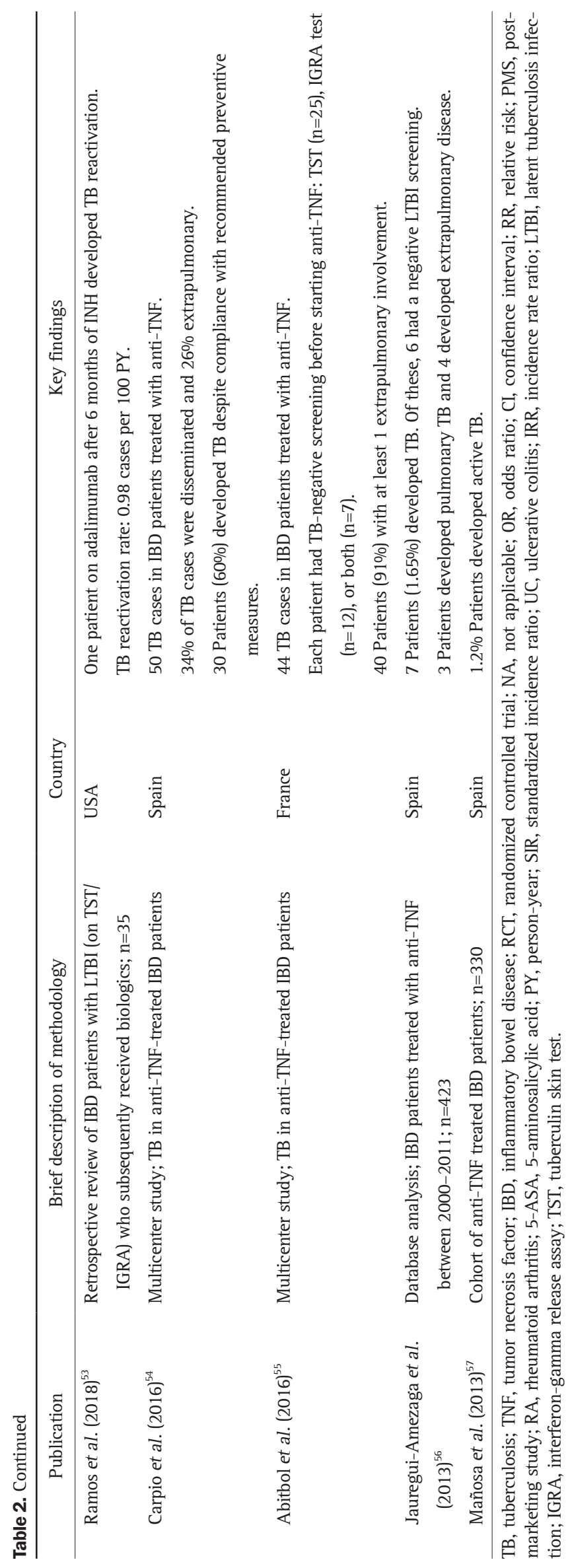




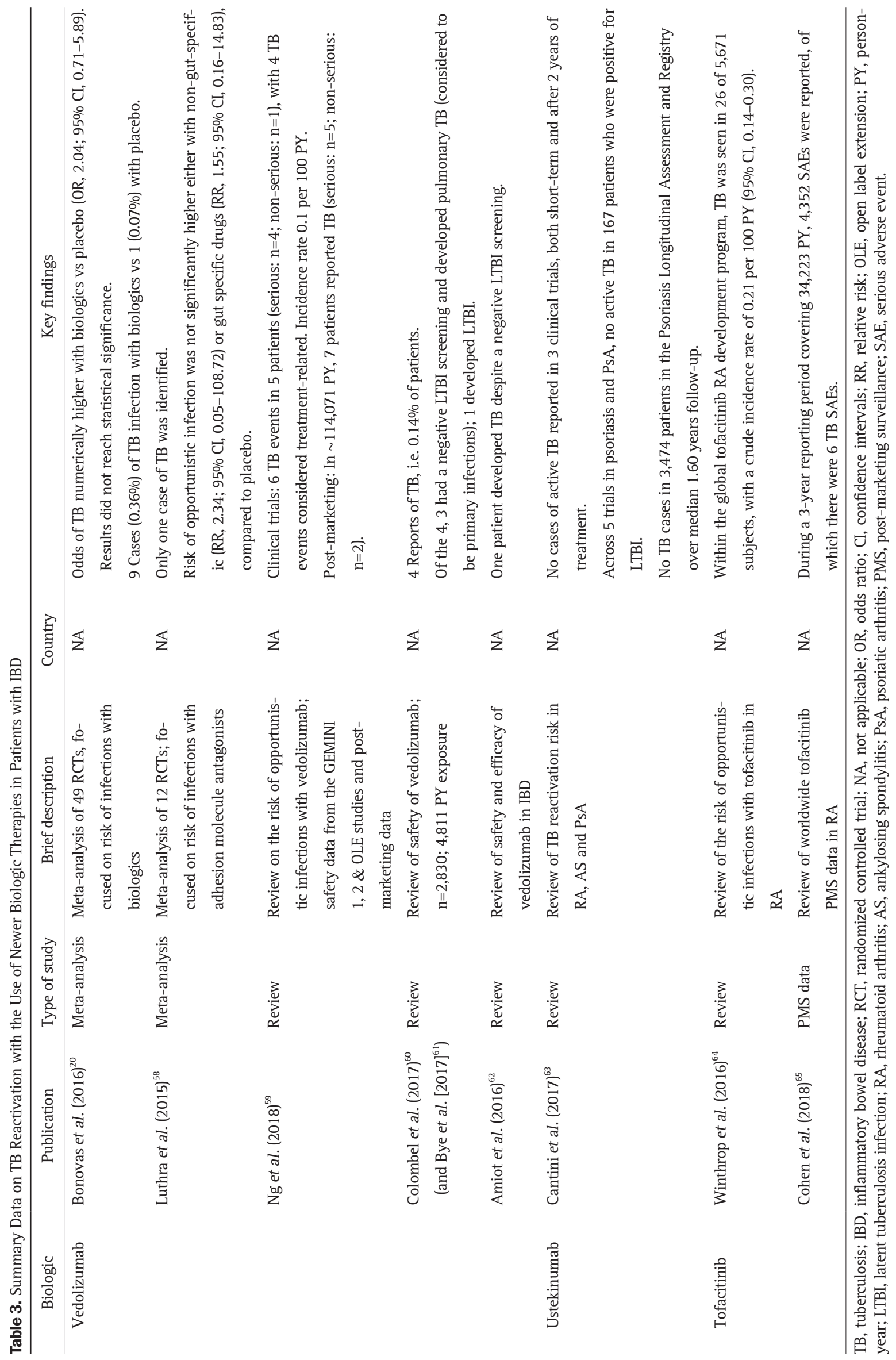




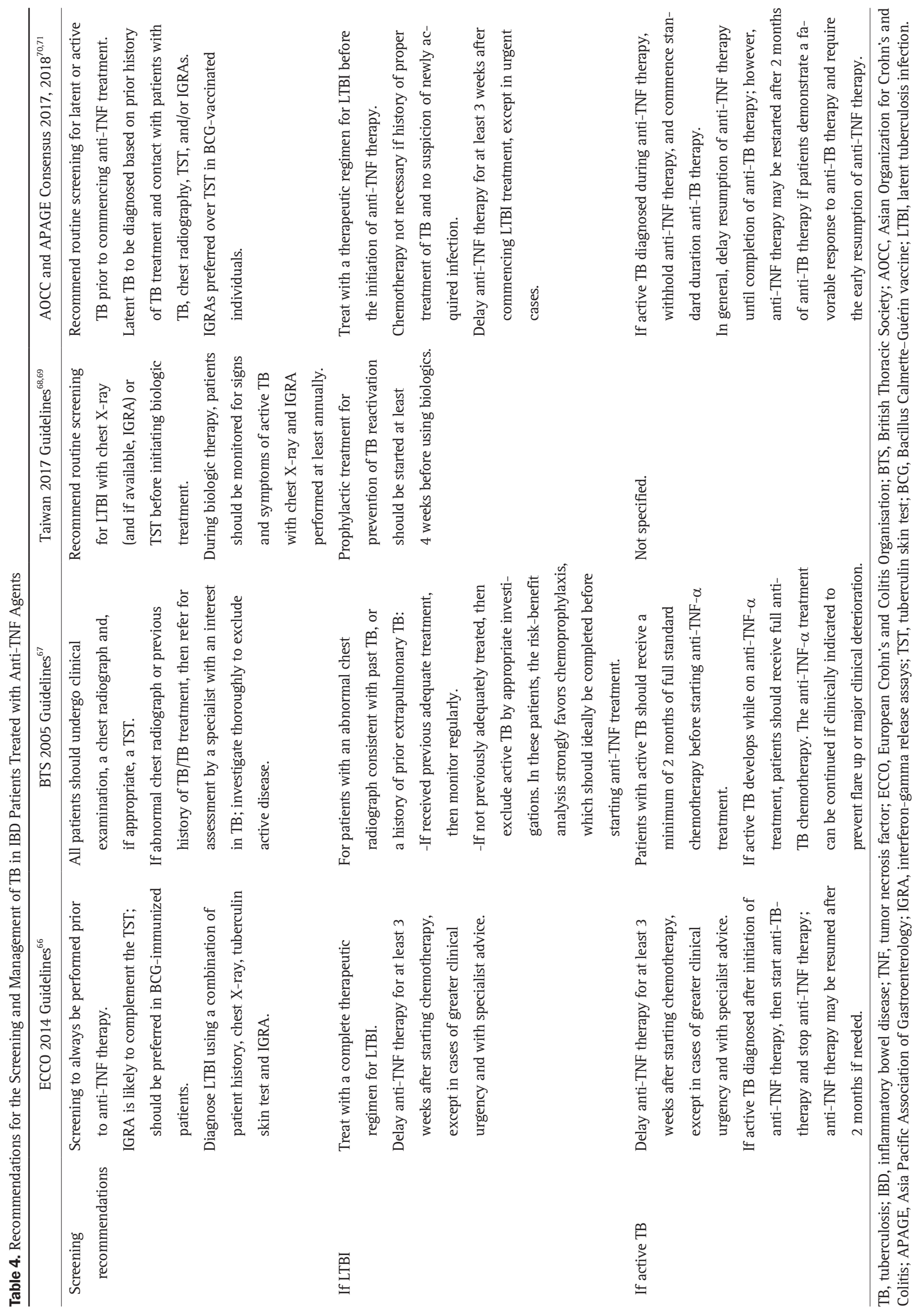


ficity of TST decreases if BCG vaccination is administered after infancy or it is administered on multiple occasions (i.e., use of booster shots). In contrast, the specificity of IGRA is independent of BCG vaccination. ${ }^{36}$

Importantly, the sensitivity of these tests is lower in patients who are receiving immune-suppressive therapy, and false negative results with the IGRA increase with an increase in immunosuppression. ${ }^{35,37}$

It is recommended to routinely perform at least a chest X-ray and TST or IGRA prior to initiating biologic therapy in patients with IBD. In clinical practice, clinicians perform screening based on availability of tests at their centers.

\section{TB REACTIVATION WITH BIOLOGICS}

\section{TB reactivation in IBD prior to the biologics era}

It should be noted that an association between IBD and TB existed even in the era prior to the availability of biologics. Aberra et $a .^{38}$ performed a retrospective cohort study with the General Practice Research Database in the United Kingdom from January 1988 to October 1997, and found that the annual incidence of active TB was significantly higher in those with IBD than controls. The unadjusted relative risk for active TB was 2.36 (95\% confidence intervals [CI], 1.17 to 4.74); after adjusting for confounders, corticosteroid use and smoking, the odds ratio failed to meet statistical significance at 1.88 (95\% CI, 0.68 to 5.20), suggesting that the increased risk was due to the combination of IBD and immunosuppressants. Thus, in the preinfliximab era, patients with IBD and immunosuppressants were associated with more than twice the risk of active TB than those without IBD. ${ }^{38}$

\section{TB reactivation with anti-TNF agents}

The issue of TB reactivation with anti-TNF agents has received considerable attention in the literature. Data from systematic reviews of the literature and recent real-world data are summarized in Table $2 .^{20,21,39-57}$ In summary: (1) meta-analyses have shown that the risk of TB in IBD patients treated with antiTNF agents is 2 to 2.5 times the risk with placebo. ${ }^{20,21}$ Although these results were not statistically significant due to the small number of cases involved, they supported the larger body of literature on the risk of TB with anti-TNF agents; (2) in the real world, $1 \%$ to $3 \%$ of IBD patients receiving treatment with antiTNF agents develop active TB; (3) worryingly, in a substantial proportion of cases, TB develops despite a negative screening test. Data on TB reactivation with anti-TNF agents in IBD and other rheumatological diseases have been reported from many Asian countries (India, Japan, Malaysia, Philippines, South Korea, Taiwan and Turkey). The risk of TB is higher with anti-TNF agents than with other treatments in IBD such as 5-ASA, corticosteroids and immunomodulators; or (4) within the anti-TNF agents, the risk of TB appears to be the highest with infliximab, followed by adalimumab.

Within auto-immune diseases, the risk of TB with anti-TNF agents may be higher in IBD than other rheumatological and dermatological diseases. This increased risk of TB in IBD appears to be driven by the higher use of infliximab compared to etanercept in these patients, though concomitant use of immunosuppressive medications may also play a role. ${ }^{39}$ Most TB cases associated with the use of anti-TNF agents in IBD manifest in the form of disseminated or military TB.

\section{TB reactivation with newer biologics-anti-integrins/ adhesion molecule antagonists/anti-IL antibodies/JAK inhibitors}

Data regarding the risk of TB reactivation with newer biologics are summarized in Table 3. ${ }^{20,58-65}$

In summary: (1) compared to data for anti-TNF agents, the available data for newer biologics are understandably limited; (2) the available data in IBD mostly pertain to vedolizumab, a gut-selective antibody to $\alpha 4 \beta 7$ integrin; (3) overall, very few cases of TB with vedolizumab have been reported: (a) in clinical trials, few cases of TB have been reported with vedolizumab and (b) similarly, in post-marketing data, the risk of TB with vedolizumab appears to be low (seven cases of TB in more than 110,000 patient years of exposure); (4) no data are available on the comparison between anti-TNF agents and adhesion molecule antagonists with reference to TB risk; or (5) data for ustekinumab and tofacitinib are available in non-IBD indications, and suggest a low risk of $\mathrm{TB}$ with the treatments in these conditions.

Although very limited, these data are suggestive of potentially lower risk of TB with adhesion molecule antagonists than antiTNF agents. This is broadly consistent with corresponding data on opportunistic infections in IBD. ${ }^{58}$

\section{Recommendations for screening and management of TB in IBD}

In view of the risk of reactivation of LTBI, routine screening for TB is recommended prior to initiation of treatment with antiTNF agents. Table 4 summarizes the recommendations from the ECCO, BTS, TSIBD, AOCC and APAGE for screening and management of TB. ${ }^{66-71}$ Note that the BTS guidelines were published in 2005 and do not discuss the role of IGRA for TB screening.

First, all guidelines recommend screening to be performed prior to initiating treatment with an anti-TNF agent in IBD. Screening should include chest X-ray and TST. The use of IGRA is recommended, especially in BCG-immunized patients. The TSIBD guidelines also recommend that patients receiving antiTNF agents should continue to be monitored for active TB with annual chest X-ray and IGRA. Second, the guidelines recommend that patients with LTBI receive standard chemotherapy for TB, which should be started at least 3 to 4 weeks before initiating treatment with anti-TNF agents. Third, active TB may 
be diagnosed before initiating treatment with anti-TNF agents or while anti-TNF treatment is ongoing. In either case, the patients should immediately receive chemotherapy for TB. The guidelines generally recommend that anti-TNF agents should not be administered during the initial 1 to 2 months of TB treatment (i.e., anti-TNF treatment should be temporarily suspended if needed); however, exceptions may be made in case of clinical urgency.

\section{DISCUSSION AND CONCLUSIONS}

In summary, TB poses many issues and challenges to clinicians in terms of diagnosis and management of IBD, especially in TB-endemic countries.

TB has diagnostic implications due to the similarities between $\mathrm{CD}$ and giTB. The difficulty in confirming the diagnosis can result in a delay in initiation of treatment. This is particularly relevant in TB-endemic countries where giTB is still a common disease and therefore needs to be suspected and ruled out wherever relevant. Unfortunately, the currently available tests are not sufficiently adequate for this purpose. While the IGRA analyses are a step forward from the previous diagnostic options, the sensitivity of the tests is not sufficiently high, resulting in false negatives. Additionally, these tests may be costly and may not always be easily accessible.

A more significant impact of $\mathrm{TB}$ is with reference to treatment with anti-TNF agents, which are a key component of the management of IBD. Inhibition of TNF- $\alpha$ can result in reactivation of latent $\mathrm{TB}$, or increase the risk of acquiring primary $\mathrm{TB}$ infections. This risk appears to be well established and has been demonstrated in clinical trials as well as in the real world. Indeed, anti-TNF agents carry a black-box warning issued by the USFDA in view of the risk of serious infections, including TB. ${ }^{72}$

Although LTBI screening with TST/IGRA helps identify some patients at risk of TB infections, a small but not insignificant proportion of results are false negatives. This problem is not easily mitigated as most IBD patients receive some form of immunosuppressive treatment, which further increases the risk of false negativity on the TST and IGRA.

In TB-endemic countries, this risk of $\mathrm{TB}$ reactivation has considerable implications for IBD management. All IBD patients need to be screened for TB prior to initiation of anti-TNF treatment. Additionally, continuous vigilance is needed while treatment with anti-TNF agents is ongoing. Also, there is currently no single guideline providing detailed TB-related recommendations for monitoring IBD patients receiving biologic treatmentsuch recommendations would need to cover, for example, the frequency of performing chest X-rays, the value of repeat IGRA while the patients are receiving anti-TNF agents, the interpretation of a normal chest X-ray with positive IGRA, guidelines regarding clinical examination to check for palpable lymphadenopathy etc. This adds an additional layer of complexity to the management of IBD in these countries.

In this context, data suggesting that the risk of TB reactivation in IBD may be lesser with anti-integrins such as vedolizumab are of interest. Although the data are currently not sufficient to allow any definitive conclusions, they are biologically plausible. Treatments not involving inhibition of TNF- $\alpha$ (which is involved in the immune reaction against TB) would be expected to be less likely to reactivate latent TB or to increase the risk of acquiring primary $\mathrm{TB}$. The gut-selective mechanism of action of vedolizumab is also very promising in this regard.

Besides the high prevalence of $\mathrm{TB}$, another issue of importance in Asia is the increasing prevalence of type 2 diabetes mellitus and obesity. ${ }^{73}$ Diabetes itself results in immune suppression thus potentially magnifying the immune-related side effects of anti-TNF agents. The presence of obesity also impacts treatment costs, as the dose of infliximab is weight based. Importantly, the presence of obesity is related to poorer response with anti-TNF agents-a recent meta-analysis showed that patients with obesity had 60\% higher odds of treatment failure with anti-TNF agents. In this analysis, although the association of obesity with inferior response did not reach statistical significance for IBD (probably due to issues with patient selection and imprecise reporting of data in the included IBD studies), the relationship showed the same trend-in IBD, patients with obesity had 20\% higher odds of failing therapy with anti-TNF agents. ${ }^{74}$ Assessing trough level of anti-TNF agents, while recommended, results in an additional cost burden. ${ }^{75}$ Other issues with the use of anti-TNF agents include the development of auto-antibodies, paradoxical immune-mediated inflammation and inefficacy due to loss of the drug in feces, especially in case of severe colonic disease. $^{76}$

Vedolizumab appears to be a promising option for the management of IBD, especially in TB-endemic regions such as the Asia-Pacific. The benefit-risk profile of vedolizumab makes it suitable for treatment-naïve patients as well as those who may require switching from anti-TNF agents to another biologic drug. Therefore, vedolizumab could potentially have a role as a first-line biologic agent for management of IBD when TB is a concern in clinical management.

Additional real-world research will help to further characterize the risk of $\mathrm{TB}$ reactivation with adhesion molecule antagonists, as well as with anti-IL-23/IL-12 agents such as ustekinumab. In order to fully understand the differential risk of $\mathrm{TB}$ reactivation between the main classes of medications, adequately powered comparisons for risk of TB reactivation between anti-TNF agents and adhesion molecule antagonists are needed. Further research is also needed to determine the most suitable screening algorithm for LTBI prior to anti-TNF treatment and to guide the plan for monitoring IBD patients receiving biologic treatments in order to prevent reactivation of TB. Given the humanistic and economic burden associated with $\mathrm{TB}$, it will be of value to conduct cost-effectiveness analysis for 
anti-TNF agents versus adhesion molecule antagonists, with particular reference to TB risk in Asian countries.

\section{CONFLICTS OF INTEREST}

S.A. is an employee of Takeda Pharmaceuticals International AG, Zurich, Switzerland. No other potential conflict of interest relevant to this article was reported.

\section{ACKNOWLEDGEMENTS}

Medical writing support was provided by Assansa, India (a Healthcare Consultancy-Assansa consultants Dr. Aamir Shaikh MD, Dr. Saifuddin Kharawala MBBS, DPM) and sponsored by Takeda Pharmaceuticals Company Ltd.

\section{ORCID}

Rupa Banerjee

Raja Affendi Raja Ali

Shu Chen Wei

Shashi Adsul

\section{REFERENCES}

1. Podolsky DK. Inflammatory bowel disease. N Engl J Med 2002;347:417-429.

2. Bernstein CN, Eliakim A, Fedail S, et al. World Gastroenterology Organisation Global Guidelines Inflammatory Bowel Disease: update august 2015. J Clin Gastroenterol 2016;50:803-818.

3. Ananthakrishnan AN. Epidemiology and risk factors for IBD. Nat Rev Gastroenterol Hepatol 2015;12:205-217.

4. Silverberg MS, Satsangi J, Ahmad T, et al. Toward an integrated clinical, molecular and serological classification of inflammatory bowel disease: report of a Working Party of the 2005 Montreal World Congress of Gastroenterology. Can J Gastroenterol 2005;19 Suppl A:5A-36A.

5. Satsangi J, Silverberg MS, Vermeire S, Colombel JF. The Montreal classification of inflammatory bowel disease: controversies, consensus, and implications. Gut 2006;55:749-753.

6. Lichtenstein GR, Hanauer SB, Sandborn WJ; Practice Parameters Committee of American College of Gastroenterology. Management of Crohn's disease in adults. Am J Gastroenterol 2009;104:465483.

7. Magro F, Gionchetti P, Eliakim R, et al. Third European evidencebased consensus on diagnosis and management of ulcerative colitis: part 1: definitions, diagnosis, extra-intestinal manifestations, pregnancy, cancer surveillance, surgery, and ileo-anal pouch disorders. J Crohns Colitis 2017;11:649-670.

8. Löwenberg M, D’Haens G. Next-generation therapeutics for IBD. Curr Gastroenterol Rep 2015;17:21.

9. Cheon JH. Understanding the complications of anti-tumor necro- sis factor therapy in East Asian patients with inflammatory bowel disease. J Gastroenterol Hepatol 2017;32:769-777.

10. Pithadia AB, Jain S. Treatment of inflammatory bowel disease (IBD). Pharmacol Rep 2011;63:629-642.

11. National Institute for Health and Care Excellence (NICE). Crohn's disease: management in adults, children and young people. NICE guideline (CG 152) [Internet]. London: NICE; c2012 [cited 2018 Sep 1]. Available from: https://www.nice.org.uk/guidance/cg152.

12. National Institute for Health and Care Excellence (NICE). Ulcerative colitis: management in adults, children and young people. NICE guideline (CG 166) [Internet]. London: NICE; c2013 [cited 2018 Sep 1]. Available from: https://www.nice.org.uk/guidance/ $\operatorname{cg} 166$.

13. Gomollón F, Dignass A, Annese V, et al. 3rd European evidencebased consensus on the diagnosis and management of Crohn's disease 2016: part 1: diagnosis and medical management. J Crohns Colitis 2017;11:3-25.

14. Harbord M, Eliakim R, Bettenworth D, et al. Third European evidence-based consensus on diagnosis and management of ulcerative colitis: part 2: current management. J Crohns Colitis 2017;11:769-784.

15. Devlin SM, Panaccione R. Evolving inflammatory bowel disease treatment paradigms: top-down versus step-up. Med Clin North Am 2010;94:1-18.

16. Rogler G. Top-down or step-up treatment in Crohn's disease? Dig Dis 2013;31:83-90.

17. Ungar B, Kopylov U. Advances in the development of new biologics in inflammatory bowel disease. Ann Gastroenterol 2016;29:243-248.

18. Zheng MK, Shih DQ, Chen GC. Insights on the use of biosimilars in the treatment of inflammatory bowel disease. World J Gastroenterol 2017;23:1932-1943.

19. U.S. Food and Drug Administration (USFDA). FDA approves new treatment for moderately to severely active ulcerative colitis [Internet]. Silver Spring: USFDA; c2018 [cited 2018 May 30]. Available from: https://www.fda.gov/NewsEvents/Newsroom/PressAnnouncements/ucm609225.htm.

20. Bonovas S, Fiorino G, Allocca M, et al. Biologic therapies and risk of infection and malignancy in patients with inflammatory bowel disease: a systematic review and network meta-analysis. Clin Gastroenterol Hepatol 2016;14:1385-1397.

21. Ford AC, Peyrin-Biroulet L. Opportunistic infections with antitumor necrosis factor- $\alpha$ therapy in inflammatory bowel disease: meta-analysis of randomized controlled trials. Am J Gastroenterol 2013;108:1268-1276.

22. Dulai PS, Siegel CA. The risk of malignancy associated with the use of biological agents in patients with inflammatory bowel disease. Gastroenterol Clin North Am 2014;43:525-541.

23. Onal IK, Kekilli M, Tanoglu A, Erdal H, Ibis M, Arhan M. Tuberculosis and Crohn's disease revisited. J Coll Physicians Surg Pak 2015;25:443-448.

24. Amarapurkar DN, Patel ND, Rane PS. Diagnosis of Crohn's disease 
in India where tuberculosis is widely prevalent. World J Gastroenterol 2008;14:741-746.

25. Weng MT, Wei SC, Lin CC, et al. Seminar report from the 2014 Taiwan Society of Inflammatory Bowel Disease (TSIBD) Spring Forum (May 24th, 2014): Crohn's disease versus intestinal tuberculosis infection. Intest Res 2015;13:6-10.

26. Banerjee R, Pal P, Girish BG, Reddy DN. Risk factors for diagnostic delay in Crohn's disease and their impact on long-term complications: how do they differ in a tuberculosis endemic region? Aliment Pharmacol Ther 2018;47:1367-1374.

27. Mack U, Migliori GB, Sester M, et al. LTBI: latent tuberculosis infection or lasting immune responses to M. tuberculosis? A TBNET consensus statement. Eur Respir J 2009;33:956-973.

28. Centers for Disease Control and Prevention (CDC). Latent tuberculosis infection: a guide for primary health care providers [Internet] CDC: Atlanta; c2013 [cited 2018 Sep 1]. Available from: https:// www.cdc.gov/tb/publications/ltbi/pdf/targetedltbi.pdf.

29. Houben RM, Dodd PJ. The global burden of latent tuberculosis infection: a re-estimation using mathematical modelling. PLoS Med 2016;13:e1002152.

30. Shea KM, Kammerer JS, Winston CA, Navin TR, Horsburgh CR Jr. Estimated rate of reactivation of latent tuberculosis infection in the United States, overall and by population subgroup. Am J Epidemiol 2014;179:216-225.

31. Murdaca G, Spanò F, Contatore M, et al. Infection risk associated with anti-TNF- $\alpha$ agents: a review. Expert Opin Drug Saf 2015;14:571-582.

32. Bruns $\mathrm{H}$, Meinken $\mathrm{C}$, Schauenberg $\mathrm{P}$, et al. Anti-TNF immunotherapy reduces $\mathrm{CD} 8+\mathrm{T}$ cell-mediated antimicrobial activity against Mycobacterium tuberculosis in humans. J Clin Invest 2009;119:1167-1177.

33. Harris J, Hope JC, Keane J. Tumor necrosis factor blockers influence macrophage responses to Mycobacterium tuberculosis. J Infect Dis 2008;198:1842-1850.

34. World Health Organization (WHO). Global tuberculosis report 2017 [Internet]. Geneva: WHO; c2018 [cited 2018 Sep 1]. Available from: https://apps.who.int/iris/handle/10665/259366.

35. Goletti D, Sanduzzi A, Delogu G. Performance of the tuberculin skin test and interferon-gamma release assays: an update on the accuracy, cutoff stratification, and new potential immune-based approaches. J Rheumatol Suppl 2014;91:24-31.

36. Pai M, Denkinger CM, Kik SV, et al. Gamma interferon release assays for detection of Mycobacterium tuberculosis infection. Clin Microbiol Rev 2014;27:3-20.

37. Ndzi EN, Nkenfou CN, Gwom LC, Fainguem N, Fokam J, Pefura Y. The pros and cons of the QuantiFERON test for the diagnosis of tuberculosis, prediction of disease progression, and treatment monitoring. Int J Mycobacteriol 2016;5:177-184.

38. Aberra FN, Stettler N, Brensinger C, Lichtenstein GR, Lewis JD. Risk for active tuberculosis in inflammatory bowel disease patients. Clin Gastroenterol Hepatol 2007;5:1070-1075.

39. Jung SM, Ju JH, Park MS, et al. Risk of tuberculosis in patients treated with anti-tumor necrosis factor therapy: a nationwide study in South Korea, a country with an intermediate tuberculosis burden. Int J Rheum Dis 2015;18:323-330.

40. Cantini F, Niccoli L, Goletti D. Adalimumab, etanercept, infliximab, and the risk of tuberculosis: data from clinical trials, national registries, and postmarketing surveillance. J Rheumatol Suppl 2014;91:47-55.

41. Tan BE, Lim AL, Kan SL, et al. Real-world clinical experience of biological disease modifying anti-rheumatic drugs in Malaysia rheumatoid arthritis patients. Rheumatol Int 2017;37:1719-1725.

42. Hong SN, Kim HJ, Kim KH, Han SJ, Ahn IM, Ahn HS. Risk of incident Mycobacterium tuberculosis infection in patients with inflammatory bowel disease: a nationwide population-based study in South Korea. Aliment Pharmacol Ther 2017;45:253-263.

43. Puri AS, Desai D, Sood A, Sachdeva S. Infliximab-induced tuberculosis in patients with UC: experience from India-a country with high prevalence of tuberculosis. J Gastroenterol Hepatol 2017;32:1191-1194.

44. Byun JM, Lee CK, Rhee SY, et al. The risk of tuberculosis in Korean patients with inflammatory bowel disease receiving tumor necrosis factor- $\alpha$ blockers. J Korean Med Sci 2015;30:173-179.

45. Byun JM, Lee CK, Rhee SY, et al. Risks for opportunistic tuberculosis infection in a cohort of 873 patients with inflammatory bowel disease receiving a tumor necrosis factor- $\alpha$ inhibitor. Scand J Gastroenterol 2015;50:312-320.

46. Çekiç C, Aslan F, Vatansever S, et al. Latent tuberculosis screening tests and active tuberculosis infection rates in Turkish inflammatory bowel disease patients under anti-tumor necrosis factor therapy. Ann Gastroenterol 2015;28:241-246.

47. Chen DY, Shen GH, Hsieh TY, Hsieh CW, Lan JL. Effectiveness of the combination of a whole-blood interferon-gamma assay and the tuberculin skin test in detecting latent tuberculosis infection in rheumatoid arthritis patients receiving adalimumab therapy. Arthritis Rheum 2008;59:800-806.

48. Takeuchi T, Tatsuki Y, Nogami Y, et al. Postmarketing surveillance of the safety profile of infliximab in 5000 Japanese patients with rheumatoid arthritis. Ann Rheum Dis 2008;67:189-194.

49. Seong SS, Choi CB, Woo JH, et al. Incidence of tuberculosis in Korean patients with rheumatoid arthritis (RA): effects of RA itself and of tumor necrosis factor blockers. J Rheumatol 2007;34:706711.

50. Kumar A. Experience with anti-tumor necrosis factor- $\alpha$ therapy in India. APLAR J Rheumatol 2006;9:136-141.

51. Navarra SV, Raso AA, Lichauco JJ, Tan PP. Clinical experience with infliximab among Filipino patients with rheumatic diseases. APLAR J Rheumatol 2006;9:150-156.

52. Thi AA, Abbara A, Bouri S, et al. Challenges in screening for latent tuberculosis in inflammatory bowel disease prior to biologic treatment: a UK cohort study. Frontline Gastroenterol 2018;9:234240.

53. Ramos GP, Stroh G, Al-Bawardy B, Faubion WA, Papadakis KA, Escalante P. Outcomes of treatment for latent tuberculosis infec- 
tion in patients with inflammatory bowel disease receiving biologic therapy. Inflamm Bowel Dis 2018;24:2272-2277.

54. Carpio D, Jauregui-Amezaga A, de Francisco R, et al. Tuberculosis in anti-tumour necrosis factor-treated inflammatory bowel disease patients after the implementation of preventive measures: compliance with recommendations and safety of retreatment. J Crohns Colitis 2016;10:1186-1193.

55. Abitbol Y, Laharie D, Cosnes J, et al. Negative screening does not rule out the risk of tuberculosis in patients with inflammatory bowel disease undergoing anti-TNF treatment: a descriptive study on the GETAID cohort. J Crohns Colitis 2016;10:1179-1185.

56. Jauregui-Amezaga A, Turon F, Ordás I, et al. Risk of developing tuberculosis under anti-TNF treatment despite latent infection screening. J Crohns Colitis 2013;7:208-212.

57. Mañosa M, Domènech E, Cabré E. Current incidence of active tuberculosis in IBD patients treated with anti-TNF agents: still room for improvement. J Crohns Colitis 2013;7:e499-e500.

58. Luthra P, Peyrin-Biroulet L, Ford AC. Systematic review and metaanalysis: opportunistic infections and malignancies during treatment with anti-integrin antibodies in inflammatory bowel disease. Aliment Pharmacol Ther 2015;41:1227-1236.

59. Ng SC, Hilmi IN, Blake A, et al. Low frequency of opportunistic infections in patients receiving vedolizumab in clinical trials and post-marketing setting. Inflamm Bowel Dis 2018;24:2431-2441.

60. Colombel JF, Sands BE, Rutgeerts P, et al. The safety of vedolizumab for ulcerative colitis and Crohn's disease. Gut 2017;66:839851.

61. Bye WA, Jairath V, Travis SPL. Systematic review: the safety of vedolizumab for the treatment of inflammatory bowel disease. Aliment Pharmacol Ther 2017;46:3-15.

62. Amiot A, Grimaud JC, Peyrin-Biroulet L, et al. Effectiveness and safety of vedolizumab induction therapy for patients with inflammatory bowel disease. Clin Gastroenterol Hepatol 2016;14:15931601.

63. Cantini F, Nannini C, Niccoli L, Petrone L, Ippolito G, Goletti D. Risk of tuberculosis reactivation in patients with rheumatoid arthritis, ankylosing spondylitis, and psoriatic arthritis receiving non-anti-TNF-targeted biologics. Mediators Inflamm 2017;2017:8909834.

64. Winthrop KL, Park SH, Gul A, et al. Tuberculosis and other opportunistic infections in tofacitinib-treated patients with rheumatoid arthritis. Ann Rheum Dis 2016;75:1133-1138.
65. Cohen S, Curtis JR, DeMasi R, et al. Worldwide, 3-year, postmarketing surveillance experience with tofacitinib in rheumatoid arthritis. Rheumatol Ther 2018;5:283-291.

66. Rahier JF, Magro F, Abreu C, et al. Second European evidencebased consensus on the prevention, diagnosis and management of opportunistic infections in inflammatory bowel disease. J Crohns Colitis 2014;8:443-468.

67. British Thoracic Society Standards of Care Committee. BTS recommendations for assessing risk and for managing Mycobacterium tuberculosis infection and disease in patients due to start antiTNF-alpha treatment. Thorax 2005;60:800-805.

68. Wei SC, Chang TA, Chao TH, et al. Management of Crohn's disease in Taiwan: consensus guideline of the Taiwan Society of Inflammatory Bowel Disease. Intest Res 2017;15:285-310.

69. Wei SC, Chang TA, Chao TH, et al. Management of ulcerative colitis in Taiwan: consensus guideline of the Taiwan Society of Inflammatory Bowel Disease. Intest Res 2017;15:266-284.

70. Park DI, Hisamatsu T, Chen M, et al. Asian Organization for Crohn's and Colitis and Asia Pacific Association of Gastroenterology consensus on tuberculosis infection in patients with inflammatory bowel disease receiving anti-tumor necrosis factor treatment: part 1: risk assessment. Intest Res 2018;16:4-16.

71. Park DI, Hisamatsu T, Chen M, et al. Asian Organization for Crohn's and Colitis and Asia Pacific Association of Gastroenterology consensus on tuberculosis infection in patients with inflammatory bowel disease receiving anti-tumor necrosis factor treatment: part 2: management. Intest Res 2018;16:17-25.

72. Lis K, Kuzawińska 0, Bałkowiec-Iskra E. Tumor necrosis factor inhibitors: state of knowledge. Arch Med Sci 2014;10:1175-1185.

73. Yoon KH, Lee JH, Kim JW, et al. Epidemic obesity and type 2 diabetes in Asia. Lancet 2006;368:1681-1688.

74. Singh S, Facciorusso A, Singh AG, et al. Obesity and response to anti-tumor necrosis factor- $\alpha$ agents in patients with select immune-mediated inflammatory diseases: a systematic review and meta-analysis. PLoS One 2018;13:e0195123.

75. Vande Casteele N, Ferrante M, van Assche G, et al. Trough concentrations of infliximab guide dosing for patients with inflammatory bowel disease. Gastroenterology 2015;148:1320-1329.

76. Brandse JF, van den Brink GR, Wildenberg ME, et al. Loss of infliximab into feces is associated with lack of response to therapy in patients with severe ulcerative colitis. Gastroenterology 2015;149:350-355. 